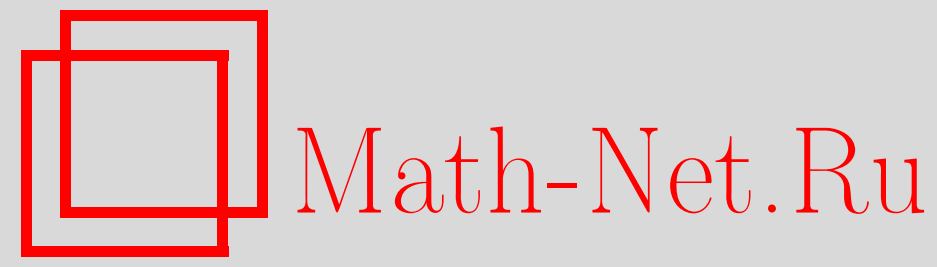

В. А. Егоров, Об асимптотическом поведении самонормированных сумм случайных величин, Теория вероятн. и ее примен., 1996, том 41, выпуск 3, 643-650

DOI: https://doi.org/10.4213/tvp3145

Использование Общероссийского математического портала Math-Net.Ru подразумевает, что вы прочитали и согласны с пользовательским соглашением http://www. mathnet.ru/rus/agreement

Параметры загрузки:

IP : 35.173 .137 .237

26 апреля 2023 г., 08:05:50

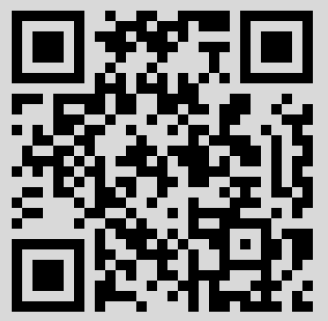


(C) $1996 \mathrm{r}$.

ETOPOB B. A.*

\title{
ОБ АСИМПТОТИЧЕСКОМ ПОВЕДЕНИИ САМОНОРМИРОВАННЫХ СУММ СЛУЧАЙНЫХ ВЕЛИЧИН ${ }^{1)}$
}

\begin{abstract}
Изучается поведение так называемых самонормированных сумм $t_{n}=S_{n} / T_{n}^{1 / 2}$, где $S_{n}=X_{1}+\cdots+X_{n}, T_{n}=X_{1}^{2}+\cdots+X_{n}^{2}, X_{i}, i=1,2, \ldots$, - симметричные независимые, но не одинаково распределенные случайные величины. Для полной и цензурированной выборок получены необходимые и достаточные условия сходимости распределения $t_{n} \mathrm{x}$ стандартному нормальному распределению.
\end{abstract}

Ключевые слова и Фразы: самонормированные суммы, симметричные независимые случайные величины, асимптотическая нормальность, относительная устойчивость, цензурированная выборка, статистика Стьюдента.

Пусть $X_{1}, X_{2}, \ldots, X_{n}$ - последовательность независимых случайных величин. Положим $S_{n}=\sum_{i=1}^{n} X_{i}, T_{n}=\sum_{i=1}^{n} X_{i}^{2}$. Давно замечено, что $S_{n}$ и $T_{n}^{1 / 2}$ ведут себя в определенном смысле синхронно. Так, в силу неравенств Марцинкевича [1] при $p>1$ величины $\mathrm{E}\left|S_{n}\right|^{p}$ и $\mathbf{E} T_{n}^{p / 2}$ грубо эквивалентны при $n \rightarrow \infty$, а в силу результатов Д. А. Райкова и Б. В. Гнеденко [2] в условиях предельной пренебрегаемости слагаемых слабый закон больших чисел для $T_{n}$ равносилен центральной предельной теореме для $S_{n}$. Поэтому естественным является интерес к асимптотическому поведению отношения $t_{n}=S_{n} / T_{n}^{1 / 2}$. Отметим, что интерес к асимптотическому поведению отношения $t_{n}$ вызван также задачами математической статистики, в которых используется статистика Стьюдента или подобные ей статистики при нарушении предположения о нормальности исходной выборки.

В этой статье исследуются условия асимптотической нормальности отношения $t_{n}$ для полной выборки и аналогичного отношения для цензурированной выборки в предположении симметричности, но не одинаковой распределенности, величин $X_{n}$. Близкие исследования для одинаково распределенных слагаемых проводились в работах [3], [7], [8].

Следующая теорема является вспомогательной, но она также представляет и самостоятельный интерес. В ней получены новые условия относительной слабой устойчивости сумм положительных независимых случайных величин.

Теорема 1. Пусть $Y_{1}, Y_{2}, \ldots, Y_{n}$ - последовательность независимых положительных случайных величин. Для того, чтобы последовательность сумм $T_{n}=$ $\sum_{i=1}^{n} Y_{i}$ была относительно устойчивой и слагаемые были бесконечно малы относительно соответствуючей нормировки, меобходмо и достаточно, чтобы выполиялось соотночение

$$
\frac{Y_{n, n}}{T_{n}} \stackrel{\mathbf{P}}{\longrightarrow} 0 \quad(n \rightarrow 0),
$$

где $Y_{n, n}=\max _{i \leqslant n} Y_{i}$. Это отночение, в свою очередь, равносильно условию

$$
\sum_{i=1}^{n} \mathbf{P}\left\{Y_{i}>\varepsilon T_{n}\right\} \longrightarrow 0 \quad(n \rightarrow \infty)
$$

для $л ю б о г о \varepsilon>0$.

*Санкт-Петербургский государственный электротехнический университет, кафедра высшей математики, ул. профессора Попова, 5, 197022 С.-Петербург, Россия.

1) Работа выполнена при поддержке Российского фонда фундаментальных исследований, грант 93-011-1459. 
3 а м е ч а н и е. Ниже мы сознательно применяем одно и то же обозначение для $\sum_{i=1}^{n} X_{i}^{2}$ и $\sum_{i=1}^{n} Y_{i}$, поскольку в последующих за этой теоремой результатах будет предполагаться, что $Y_{i}=X_{i}^{2}, i \leqslant n$.

Д о к а за т е л ь с т в о т е о р е м ы 1. Пусть выполнено соотношение (1). Введем величину $Z_{n}=\sum_{i=1}^{n} I\left\{Y_{i}>\varepsilon T_{n}\right\}$, где $I(A)$ - индикатор события $A$. Очевидно, верно неравенство

$$
0 \leqslant Z_{n} \leqslant \frac{1}{\varepsilon}
$$

Поэтому выполнено условие

$$
\mathbf{E} Z_{n}=\sum_{i=1}^{n} \mathbf{P}\left\{Y_{i}>\varepsilon T_{n}\right\} \leqslant \frac{\mathbf{P}\left\{Z_{i} \neq 0\right\}}{\varepsilon}=\frac{\mathbf{P}\left\{Y_{n, n}>\varepsilon T_{n}\right\}}{\varepsilon}
$$

Из соотношения (4) следует соотношение (2). То, что соотношение (2) влечет за собой (1), очевидно. Таким образом, соотношения (1) и (2) равносильны.

Далее, в силу условия (2) можно найти такую последовательность положительных чисел $\varepsilon_{n}, \varepsilon_{n} \downarrow 0$, для которой выполняются неравенства

$$
\sum_{i=1}^{n} \mathrm{P}\left\{Y_{i}>\varepsilon_{n} T_{n}\right\} \leqslant \varepsilon_{n} .
$$

Из (5) получаем, что

$$
\sum_{i=1}^{n} \mathbf{P}\left\{Y_{i}>\varepsilon_{n}^{\prime} T_{n, i}\right\} \leqslant \varepsilon_{n}^{\prime}
$$

где $\varepsilon_{n}^{\prime}=\varepsilon_{n} /\left(1-\varepsilon_{n}\right), T_{n, i}=T_{n}-Y_{i}$. Поэтому для любой числовой последовательности $x_{n}$ выполняются неравенства

$$
\sum_{i=1}^{n} \mathbf{P}\left\{Y_{i}>x_{n}, x_{n}>\varepsilon_{n}^{\prime} T_{n, i}\right\} \leqslant \varepsilon_{n}^{\prime}
$$

или

$$
\sum_{i=1}^{n} \mathbf{P}\left\{Y_{i}>x_{n}\right\} \mathbf{P}\left\{x_{n}>\varepsilon_{n}^{\prime} T_{n, i}\right\} \leqslant \varepsilon_{n}^{\prime} .
$$

Тем более, выполнены условия

$$
\sum_{i=1}^{n} \mathbf{P}\left\{Y_{i}>x_{n}\right\} \mathbf{P}\left\{T_{n}<\frac{x_{n}}{\varepsilon_{n}^{\prime}}\right\} \leqslant \varepsilon_{n}^{\prime}
$$

Пусть теперь числовые последовательности $\delta_{n} \downarrow 0$ и $x_{n}$ выбраны таким образом, что

$$
\frac{\delta_{n}}{\varepsilon_{n}^{\prime}} \rightarrow \infty, \quad \mathbf{P}\left\{Y_{n, n}<x_{n}\right\}=\prod_{i=1}^{n} G_{i}\left(x_{n}\right)=1-\delta_{n} \longrightarrow 1
$$

где $G_{i}(x)=\mathbf{P}\left\{Y_{i}<x\right\}$. Тогда из условия (8) следует соотношение

$$
\max _{i \leqslant n} P\left\{Y_{i}>x_{n}\right\} \longrightarrow 0 \quad(n \rightarrow \infty),
$$

и поэтому из условий (7), (8) вытекает неравенство

$$
\mathbf{P}\left\{T_{n}<\frac{x_{n}}{\varepsilon_{n}^{\prime}}\right\} \leqslant C \frac{\varepsilon_{n}^{\prime}}{\delta_{n}} \longrightarrow 0 \quad(n \rightarrow \infty),
$$


где $C>0$ есть некоторая постоянная. Из соотношений (8), (9) выводим, что

$$
\mathbf{P}\left\{T_{n}>\frac{x_{n}}{\varepsilon_{n}^{\prime}}, Y_{n, n}<x_{n}\right\} \rightarrow 1 \quad(n \rightarrow \infty)
$$

Поэтому, полагая

$$
Y_{i, n}^{\prime}=\left\{\begin{array}{lll}
Y_{i}, & \text { если } \quad Y_{i}<x_{n}, \\
0, & \text { если } \quad Y_{i} \geqslant x_{n},
\end{array} \quad T_{n}^{\prime}=\sum_{i=1}^{n} Y_{i, n}^{\prime},\right.
$$

получим соотношение

$$
\mathbf{P}\left\{T_{n}^{\prime}>\frac{x_{n}}{\varepsilon_{n}^{\prime}}\right\} \rightarrow 1 \quad(n \rightarrow \infty)
$$

С другой сторонщ, в силу неравенства Чебышева,

$$
\mathbf{P}\left\{T_{n}^{\prime}>2 \sum_{i=1}^{n} \mathbf{E} Y_{i, n}^{\prime}\right\} \leqslant \frac{1}{2} .
$$

Сравнивая соотношения (11) и (12), придем к неравенству

$$
2 \sum_{i=1}^{n} \mathrm{E} Y_{i, n}^{\prime}>\frac{x_{n}}{\varepsilon_{n}^{\prime}}
$$

Поэтому

$$
\frac{\sum_{i=1}^{n} \mathbf{E} Y_{i, n}^{\prime}}{x_{n}}=\frac{\sum_{i=1}^{n} \int_{x<x_{n}} x d G_{i}(x)}{x_{n}} \longrightarrow \infty .
$$

Кроме того, очевидно, выполняется условие

$$
\sum_{i=1}^{n} \mathbf{P}\left\{Y_{i}>x_{n}\right\} \sim \delta_{n} \longrightarrow 0 \quad(n \rightarrow \infty)
$$

В теореме 3 [2] условия (14) и (15) приведены как необходимые и достаточные условия для слабой относительной устойчивости последовательности сумм $T_{n}$.

Обратное утверждение теоремы легко вытекает из ее условия и критерия вырожденной сходимости [4] или критерия относительной слабой устойчивости [2].

Ниже для удобства ссылок предлолагается, что $Y_{i}=X_{i}^{2}, i=1,2, \ldots$.

Теорема 2. Пусть $X_{1}, X_{2}, \ldots, X_{n}, \ldots$ - последовательность независимых симметричных случайных величин. Положим

$$
\Phi(x)=\int_{-\infty}^{x} e^{-t^{2} / 2} d t, \quad \nabla_{n}=\sup _{x}\left|\mathbf{P}\left\{t_{n}<x\right\}-\Phi(x)\right| .
$$

Тогда для справедливости соотночения

$$
\nabla_{n} \rightarrow 0 \quad(n \rightarrow \infty)
$$

необходимо и достаточно, чтобы выполнялось условие (1) или эквивалектное ему условие (2).

Таким образом, центральная предельная теорема для $t_{n}$ равносильна условию бесконечной малости слагаемых и слабой устойчивости сумм $T_{n}$ и поэтому равносильна в условиях предельной пренебрегаемости слагаемых центральной предельной теореме с неслучайной нормировкой. Аналогичный результат для симметричных одинаково распределенных назависимых слагаемых получен в работе [3]. 
Доказательство теоремы 2. Н еоб ходимость. Отметим сначала, что величины $X_{n}$ остаются условно независимыми, если фиксирована последовательность $\left(Y_{n}, n>0\right)$, где $Y_{n}=X_{n}^{2}$. Этот факт использовался еше Марцинкевичем, о чем указано во вступительной статье Зигмунда сборника трудов Марцинкевича [6]. Его доказательство этого факта очень просто и коротко. Действительно, пусть на некотором вероятностном пространстве задана последовательность независимых одинаково распределенных симметричных случайных величин $\left(u_{n}, n>0\right)$, принимающих с вероятностями $\frac{1}{2}$ значения \pm 1 , а исходная последовательность случайных величин $X_{n}$ задана на другом вероятностном пространстве. На произведении этих вероятностных пространств зададим последовательность случайных величин $\left(Z_{n}, n>0\right)$, положив $Z_{n}=Y_{n}^{1 / 2} u_{n}, n=1,2, \ldots$. Легко видеть, что при фиксированных $\left(Y_{i}, i>0\right)$ случайные величины $\left(Z_{i}, i>0\right)$ независимы между собой и распределения случайных последовательностей $\left(X_{n}, n>0\right)$ и $\left(Z_{n}, n>0\right)$ совпадают. Поскольку последовательность $\left(Z_{n}, n>0\right)$ по ее построению обладает доказываемым здесь свойством, этим свойством обладает и последовательность $\left(X_{n}, n>0\right)$. Будем ниже снабжать штрихом $\left(\mathbf{E}^{\prime}, \mathbf{P}^{\prime}\right.$ и т.д.) соответствующие величины, если речь будет идти об условном распределении при фиксированных $Y_{n}, n>0$.

Пусть теперь выполнено условие (16). Тогда для любого фиксированного $t$

$$
\mathrm{E} \exp \left\{i t t_{n}\right\}-e^{-t^{2} / 2} \longrightarrow 0 \quad(n \rightarrow \infty)
$$

Используя условную относительно последовательности $Y_{n}$ независимость величин $X_{n}$, получим из (17) соотношекие

$$
\mathrm{EE}^{\prime} \exp \left\{i t t_{n}\right\}-e^{-t^{2} / 2}=\mathbf{E}\left[\prod_{j=1}^{n} \cos \left(\frac{t\left|X_{j}\right|}{T_{n}^{1 / 2}}\right)-e^{-t^{2} / 2}\right] \longrightarrow 0 .
$$

Применяя неравенство $\cos z \leqslant \exp \left(-z^{2} / 2\right)$, верное для всех достаточно малых $z^{2}$, и очевидное неравенство $\left|X_{i}\right| / T_{n}^{1 / 2} \leqslant 1$, находим, что при всех достаточно малых $t$. выполняется предельное соотношение

$$
\mathbf{E}\left|\prod_{i=1}^{n} \cos \left(\frac{t\left|X_{i}\right|}{T_{n}^{1 / 2}}\right)-e^{-t^{2} / 2}\right| \longrightarrow 0 \quad(n \rightarrow \infty) .
$$

Используя при малых $z$ разложение Маклорена функции $\ln \cos z$, получим

$$
\ln \cos z=-\frac{z^{2}}{2}-\frac{z^{4}}{12}+\theta z^{6}, \quad|\theta|<C
$$

где $C$ - постоянная. Из соотношений (19), (20) при достаточно малых $|t|$ следует соотношение

$$
\mathrm{E}\left|\exp \left\{-\frac{t^{4}}{12 T_{n}^{2}} \sum_{i=1}^{n} X_{i}^{4}+\theta \frac{t^{6}}{T_{n}^{3}} \sum_{i=1}^{n} X_{i}^{6}\right\}-1\right| \longrightarrow 0 \quad(n \rightarrow \infty) .
$$

Поэтому для всех достаточно малых $|t|$

$$
\frac{1}{T_{n}^{2}} \sum_{i=1}^{n} X_{i}^{4}+\frac{12 \theta t^{2}}{T_{n}^{3}} \sum_{i=1}^{n} X_{i}^{6} \longrightarrow 0 \quad(n \rightarrow \infty) .
$$

В силу (22),

$$
\frac{1}{T_{n}^{2}} \sum_{i=1}^{n} X_{i}^{4} \stackrel{\mathbf{P}}{\longrightarrow} 0 \quad(n \rightarrow \infty)
$$


С другой стороны, справедливо неравенство

$$
\frac{1}{T_{n}^{2}} \sum_{i=1}^{n} X_{i}^{4} \geqslant \frac{1}{T_{n}^{2}} \max _{i \leqslant n} X_{i}^{4}=\left(\frac{Y_{n, n}}{T_{n}}\right)^{2} .
$$

Из соотношений (23), (24) следует соотношение (1).

Перейдем $\mathrm{k}$ дохазательству д о с т а т о ч н о с т и. В силу теоремы Эссеена справецливо неравенство

$$
\left|\mathbf{P}^{\prime}\left\{t_{n}<x\right\}-\Phi(x)\right| \leqslant C \frac{1}{T_{n}^{3 / 2}} \sum_{i=1}^{n}\left|X_{i}^{3}\right| .
$$

Поэтому

$$
\nabla_{n} \leqslant C \mathrm{E} \frac{1}{T_{n}^{3 / 2}} \sum_{i=1}^{n}\left|X_{i}^{3}\right| \leqslant C \mathrm{E} \frac{\max _{i \leqslant n}\left|X_{i}\right|}{T_{n}^{1 / 2}} .
$$

Поскольку последнее выражение под знаком математического ожидания не превосходит единицы, из этого неравенства следует, что для любого $\varepsilon>0$

$$
\nabla_{n} \leqslant C\left(\varepsilon+\mathbf{P}\left\{\max _{i \leqslant n}\left|X_{i}\right|>\varepsilon T_{n}^{1 / 2}\right\}\right),
$$

откуда вытекает достаточность условия (1). Теорема 2 доказана.

Отметим, что в теореме 2 априори не предполагается выполненным условие предельной пренебрегаемости слагаемых.

Рассмотрим теперь цензурированные выборки. Пусть $X_{n, i}, i \leqslant n, n=1,2, \ldots$, есть абсолютные порядковые статистики, т.е. $\left|X_{n, 1}\right| \leqslant\left|X_{n, 2}\right| \leqslant \cdots \leqslant\left|X_{n, n}\right|$. Будем предполагать, что выборка цензурирована, т.е. из нее удалены элементы $X_{n, n}$, $X_{n, n-1}, \ldots, X_{n, n-k+1}$. Положим

$$
\begin{aligned}
S_{n, k} & =\sum_{j=1}^{n-k} X_{n, j}, \quad T_{n, k}=\sum_{j=1}^{n-k} X_{n, j}^{2}=\sum_{j=1}^{n-k} Y_{n, j}, \quad t_{n, k}=\frac{S_{n, k}}{\left(T_{n, k}\right)^{1 / 2}}, \\
\nabla_{n, k} & =\sup \left|\mathbf{P}\left\{t_{n, k}<x\right\}-\Phi(x)\right| .
\end{aligned}
$$

Теорема 3. Пусть $X_{1}, X_{2}, \ldots, X_{n}$ - последовательность кезависимых симметричных случайных величин. Дая того, чтобы

$$
\nabla_{n, k} \rightarrow 0 \quad(n \rightarrow \infty)
$$

меобходимо и достаточно, чтобы

$$
\frac{X_{n, n-k}^{2}}{T_{n, k}} \longrightarrow 0 \quad(n \rightarrow \infty) .
$$

Д,оказа те ль с тв о теоремы 3 аналогично доказательству теоремы 2. Поэтому мы его опускаем.

Покажем теперь, что при достаточно слабых дополнительных предположениях условия (26), а, следовательно, и условия (25), при различных фиксированных $k$ равносильны.

Ниже, как и раньше, считаем, что $Y_{n}=X_{n}^{2}$.

Введем условие предельной пренебрегаемости слагаемых в виде

$$
\max _{i \leqslant n} \mathbf{P}\left\{Y_{i}>\varepsilon T_{n}\right\} \longrightarrow 0 \quad(n \rightarrow \infty)
$$


для любого $\varepsilon>0$ и условие

$$
\limsup _{\lambda \rightarrow \infty} \sup _{x>x_{0}} \frac{\sum_{i=1}^{n} \mathbf{P}\left\{Y_{j}>\lambda x\right\}}{\sum_{j=1}^{n} \mathbf{P}\left\{Y_{j}>x\right\}}<B<1
$$

для некоторого $B$ и всех достаточно больших $n$.

Теорема 4. При выполнеяии условий (27), (28) условия (26) и (1) эквивалентMbl.

Д ок а з а т е л ь с т в о. Очевидно, из (1) вытекает (26). Покажем обратное включение. Пусть выполнены условия (26)-(28). Положим для произвольного $\varepsilon>0$

$$
Z_{n}=\sum_{i_{1}=1}^{n} \sum_{i_{2}=1}^{i_{1}-1} \cdots \sum_{i_{k+1}=1}^{i_{k}-1} I\left\{Y_{i_{1}}>\varepsilon T_{n}\right\} I\left\{Y_{i_{2}}>\varepsilon T_{n}\right\} \cdots I\left\{Y_{i_{k+1}}>\varepsilon T_{n}\right\}
$$

где $I(A)-$ индикатор события $A$. Очевидно, $Z_{n}<1 / \varepsilon^{k+1}$. Поэтому

$$
\begin{aligned}
\mathbf{E} Z_{n} & =\sum_{i_{1}=1}^{n} \sum_{i_{2}=1}^{i_{1}-1} \cdots \sum_{i_{k+1}=1}^{i_{k}-1} \mathbf{P}\left\{Y_{i_{1}}>\varepsilon T_{n}, Y_{i_{2}}>\varepsilon T_{n}, \ldots, Y_{i_{k+1}}>\varepsilon T_{n}\right\} \\
& \leqslant \frac{\mathbf{P}\left\{Z_{n}>0\right\}}{\varepsilon^{k+1}}=\frac{\mathbf{P}\left\{Y_{n, n-k}>\varepsilon T_{n}\right\}}{\varepsilon^{k+1}} \longrightarrow 0 .
\end{aligned}
$$

Положим

$$
\begin{aligned}
A\left(i_{1}, i_{2}, \ldots, i_{k+1}\right) & =Y_{i_{1}}+Y_{i_{2}}+\cdots+Y_{i_{k+1}}, \\
A^{i}\left(i_{1}, i_{2}, \ldots, i_{k+1}\right) & =A\left(i_{1}, i_{2}, \ldots, i_{k+1}\right)-Y_{i}, \\
B\left(i_{1}, i_{2}, \ldots, i_{k+1}\right) & =T_{n}-A\left(i_{1}, i_{2}, \ldots, i_{k+1}\right) .
\end{aligned}
$$

Тогда из соотношений (29) следует, что найдется такая последовательность $\varepsilon_{n}$, достаточно медленно сходяшаяся $\mathbf{k}$ нулю, что

$$
\begin{gathered}
\sum_{i_{1}=1}^{n} \sum_{i_{2}=1}^{i_{1}-1} \ldots \sum_{i_{k+1}=1}^{i_{k}-1} \mathbf{P}\left\{Y_{i_{1}}-\varepsilon_{n} A^{i_{1}}\left(i_{1}, i_{2}, \ldots, i_{k+1}\right)>\varepsilon_{n} B\left(i_{1}, i_{2}, \ldots, i_{k+1}\right), \ldots,\right. \\
Y_{i_{k+1}}-\varepsilon_{n} A^{i_{k+1}\left(i_{1}, i_{2}, \ldots, i_{k+1}\right)} \\
\left.>\varepsilon_{n} B\left(i_{1}, i_{2}, \ldots, i_{k+1}\right)\right\}<\varepsilon_{n}^{k+1} .
\end{gathered}
$$

Поэтому с учетом неравенства $B\left(i_{1}, i_{2}, \ldots, i_{k+1}\right) \leqslant T_{n}$ получим, что для любой последовательности $x_{n}>0$ справедливо неравенство

$$
\begin{array}{rl}
\sum_{i_{1}=1}^{n} \sum_{i_{2}=1}^{n} \ldots \sum_{i_{k+1}=1}^{n} & \mathbf{P}\left\{Y_{i_{1}}-\varepsilon_{n} A^{i_{1}}\left(i_{1}, i_{2}, \ldots, i_{k+1}\right)>x_{n}, \ldots,\right. \\
\left.Y_{i_{k+1}}-\varepsilon_{n} A^{i_{k+1}}\left(i_{1}, i_{2}, \ldots, i_{k+1}\right)>x_{n}\right\} \\
\times \mathbf{P}\left\{T_{n}<\frac{x_{n}}{\varepsilon_{n}}\right\}<\varepsilon_{n}^{k+1} .
\end{array}
$$

Далее, так как $A^{i_{j}}\left(i_{1}, i_{2}, \ldots, i_{k+1}\right) \leqslant k \lambda_{n} x_{n}$, если $Y_{i_{j}} \leqslant \lambda_{n} x_{n}$ для всех $j=1,2, \ldots, k+$ 1 , то для ліюбой последовательности $\lambda_{n}>0$

$$
\begin{array}{r}
\mathbf{P}^{k+1}\left\{T_{n}<\frac{x_{n}}{\varepsilon_{n}}\right\} \sum_{i_{1}=1}^{n} \sum_{i_{2}=1}^{i_{1}-1} \cdots \sum_{i_{k+1}=1}^{i_{k}-1} \mathbf{P}\left\{x_{n}\left(1+k \varepsilon_{n} \lambda_{n}\right)<Y_{i_{j}}<\lambda_{n} x_{n}\right. \\
j=1,2, \ldots, k+1\}<\varepsilon_{n}^{k+1}
\end{array}
$$


илли

$$
\begin{aligned}
& \mathbf{P}^{k+1}\left\{T_{n}<\frac{x_{n}}{\varepsilon_{n}}\right\} \\
& \quad \times \sum_{i_{1}=1}^{n} \sum_{i_{2}=1}^{i_{1}-1} \ldots \sum_{i_{k+1}=1}^{i_{k}-1} \prod_{j=1}^{k+1} \mathbf{P}\left\{x_{n}\left(1+k \varepsilon_{n} \lambda_{n}\right)<Y_{i_{j}}<\lambda_{n} x_{n}\right\}<\varepsilon_{n}^{k+1}
\end{aligned}
$$

Пусть $\varepsilon_{n} \lambda_{n} \rightarrow 0$. Тогда, используя условие (27) и рассуждения, аналогичные приведенным выше, получим, что найдется такая последовательность $\varepsilon \downarrow 0$, которую, не умаляя общности, можно взять равной последовательности из (32), что

$$
\max _{i \leqslant n} \mathbf{P}\left\{T_{n}<\frac{x_{n}}{\varepsilon_{n}}\right\} \mathbf{P}\left\{x_{n}\left(1+k \varepsilon_{n} \lambda_{n}\right)<Y_{\mathbf{i}}<\lambda_{n} x_{n}\right\} \leqslant \varepsilon_{n} .
$$

Нам далее потребуется следующая лемма, доказанная в [5].

Лемма. Пусть $Y_{j}$ - последовательность положительных иисел,

$$
Q_{k, n}=\sum Y_{j_{1}} Y_{j_{2}} \cdots Y_{j_{k}}
$$

әде суммирование ведется по всем индексам при условии $1 \leqslant j_{1}<j_{2}<\cdots<j_{k} \leqslant$ n. При любых челых положитеяьных $п$ и $р$ и при любом положительмом $A$, если $Y_{j} \leqslant A, j \leqslant n, u Q_{p, n} \leqslant A^{p}$, mo $\sum Y_{j} \leqslant(2 p-1) A$.

Применяя лемму, из неравенств (32), (33) получаем

$$
\mathbf{P}\left\{T_{n}<\frac{x_{n}}{\varepsilon_{n}}\right\} \sum_{i=1}^{n} \mathbf{P}\left\{x_{n}\left(1+k \varepsilon_{n} \lambda_{n}\right)<Y_{i}<\lambda_{n} x_{n}\right\}<(2 k+1) \varepsilon_{n} .
$$

Выберем последовательность $\lambda_{n}$ так, чтобы $\varepsilon_{n} \lambda_{n} \rightarrow 0, \lambda_{n} \rightarrow \infty$, иусть $x_{n} \rightarrow \infty$. Тогда, используя условие (28), из (34) выводим

$$
\mathbf{P}\left\{T_{n}<\frac{x_{n}}{\varepsilon_{n}}\right\} \sum_{j=1}^{n} \mathbf{P}\left\{Y_{j}>x_{n}\left(1+k \varepsilon_{n} \lambda_{n}\right)\right\} \leqslant(2 k+1) \varepsilon_{n} .
$$

Последнее неравенство практически совпадает с соотношением (7). Поэтому последняя часть доказательства совпадает с соответствующей частью доказательства теоремы 1 . Следовательно, последовательность $T_{n}$ слабо устойчива и выполнено соотношение (1).

\section{СПИСОК ЛИТЕРАТУРЫ}

1. Marcinkiewicz J., Zygmund A. Sur les fonction independentes. - Fund. Math., 1937, v. 29, p. $60-90$.

2. Гнедекко Б. B., Колмогоров $A$. Н. Предельные распределения для сумм независимых случайных величин. М.-Л.: Гостехиздат, 1949.

3. Maller $R$. A. Asymptotic normality of lightly trimmed means - a converse. - Math. Proc. Cambridge Philos. Soc., 1982, v. 92, № 3, p. 535-545.

4. Лоэв М. Теория вероятностей. М.: ИЛ, 1962.

5. Егоров $B . A$. Об усиленном законе больших чисел и законе повторного логарифма для последовательности независимых случайных величин. - Теория вероятн. и ее примен., 1970, т. 15 , в. 3, с. 520-528.

6. Zygmund A. Josef Marcinkiewicz, Collected papers. Warszawa: Panstwowe wydawnictwo naukowe, 1964. 
7. Hahn M. G., Kuelbs J., Weiner D. C. The asymptotic joint distribution of selfnormalized censored sums and sums of squares. - Ann. Probab., 1990, v. 18, № 3, p. 1284-1341.

8. Hahn M. G., Kuelbs J., Weiner D. S. The asymptotic distribution of magnitude -Winsorized sums via self-normalization. - J. Theoret. Probab., 1990, № 1, p. 137168.

Поступила в редакцию 10.II.1994

\section{(c) 1996 г. КАIIТАНОВ В. А.*, ЯНИШЕВСКИЙ И. М.** \\ COBMECTHOE РАСПРЕДЕЛЕНИЕ MOMEHTA КАTACTРОФЫ И АДДИТИВНОГО ФУНКЦИОНАЛА ДОХОДОВ}

В работе определяется модель управляемого полумарковского процесса $\mathrm{c}$ катастрофами, обобщающая исследованную А. Д. Соловьевым модель процесса восстановления с катастрофами. Исследуется двумерный вектор, каждая компонента которого является аддитивным функционалом, построенным на траекториях этого процесса. Получено уравнение для совместного распределения этих функционалов и доказаны теоремы, описываюшие структуру математических ожиданий и смешанного момента в зависимости от функций распределения, определяющих стратегию управления.

Ключевые слова и Фразы: управляемый полумарковский процесс, катастрофа, аддитивный функциоал, двумерный смешанный момент, структура функционала, стратегкя управления.

Процесс марковского восстановления $\left\{\xi_{n}, \theta_{n}, n \geqslant 0\right\}$ со значениями в $\mathbb{E} \times \mathbf{R}^{+}(\mathbb{E}-$ конечное множество) является исходным объектом для конструктивного задания семейства полумарковских процессов специального типа. Общепринито, что первая компонента образует однородную цепь Маркова, фиксирующую состояние процесса на $n$-м шаге. Вторая компонента $\theta_{n}$ фиксирует время пребынания в состоянии $\xi_{n}$. Пусть на каждом шаге процесса марковского восстановления происходит некоторое событие $A_{n}$. Определим случайную величину $\eta_{n}$ как интервал времени от $n$-го скачка прощесса до наступления события $A_{n}$. Далее, предположим, что процесс марковского восстановления управляется в моменты изменения состояния выбором решения $\nu_{n}$ со значениями в измеримом пространстве $(U, \mathfrak{U})$, где $U$ интерпретируется как множество управлений, а $\sigma$-алгебра $\mathfrak{U}$-измеримых множеств из $U$ (предполагается, что она содержит одноточечные подмножества) интерпретируется как совокупность подмножеств управлений. Добавим к базовой последовательности две компоненты $\eta_{n}$ и $\nu_{n}$, $n \geqslant 0$. Такая последовательность $\left\{\xi_{n}, \theta_{n}, \eta_{n}, \nu_{n}, n \geqslant 0\right\}$ является однородной цепью Маркова, которая задается начальным распределением $\mathbf{P}\left\{\xi_{0}=e_{i}\right\}=p_{i}, e_{i} \in \mathbb{E}$, и переходными вероятностями, определяемыми ядром

$$
\mathbf{P}\left\{\xi_{n}=e_{j}, \theta_{n}<x, \eta_{n}<y, \nu_{n} \in U \mid \xi_{n-1}=e_{i}\right\}=D_{i j}(x, y, U) .
$$

*Московский государственный институт математики и электроники, Б. Трехсвятительский пер., $3 / 12$, Москва, Россия.

** Институт высокопроизводительных вычислительных систем РАН, ул. Вавилова, 39, Москва, Россия. 